\title{
Guided Mental Practice: \\ An Instructional Strategy for Asynchronous e-Learning of Pilot Safety Skills
}

Suzanne K. Kearns, Ph.D.

University of Western Ontario

\begin{abstract}
This investigation assessed guided mental practice (GMP), a new practice approach for online learning, within a pilot safety training program. With GMP learners view a video of a flight simulator scenario and imagine themselves as the pilot of the flight. Thirty six licensed pilots participated in the study and were randomly separated into three groups: (a) training with hands-on practice facilitated through yoke and rudder pedal-controlled flight scenarios, (b) training with GMP where participants observed a flight scenario video, and (c) a control group that did not receive any training. Post-training, pilot participants completed a cross country flight in a high-fidelity simulator. During the simulator flight, each participant's situation awareness was assessed. The results of the study suggest that training incorporating GMP can be equally effective as training with hands-on practice in improving pilot safety performance.
\end{abstract}

\section{INTRODUCTION}

To address the large number of human error accidents in aviation, most airline and military pilots are required to complete annual safety training known as crew resource management (CRM) (Jensen, 1995). There are several concepts included in CRM training, such as communication and coordination with crewmembers, aeronautical decision making, automation management, workload management, and situation awareness. A recent variation of CRM is single-pilot resource management (SRM) which adapts CRM concepts for use in single-pilot operations. Unlike CRM which is primarily designed for airline and military pilots, SRM is appropriate for general aviation pilots who may not otherwise have access to advanced safety training.

In airline and military environments, CRM concepts are taught in a classroom and followed by practice in a high-fidelity flight simulator (Helmreich, Merritt, \& Wilhelm, 1999). The disadvantage to this approach is that high-fidelity flight simulators are extremely expensive to operate, large training facilities must be maintained, and pilots must be pulled from active duty to complete training. However, simulator practice of CRM concepts is important as a lack of practice opportunities can result in pilots developing declarative knowledge but lacking the procedural knowledge required to apply the skills outside of the classroom to novel situations (Salas, Rhodenizer, \& Bowers, 2000). Integrating practice into SRM training is problematic, as high-fidelity simulators are prohibitively expensive or inaccessible to many GA pilots. However, since practice remains an important element of training, a method of incorporating practice within SRM is required. The delivery of CRM and SRM via e-learning can ameliorate many these issues as it provides complete temporal and geographic flexibility at minimal cost. In addition, low-fidelity simulation may be integrated into e-learning as a method of practicing CRM or SRM concepts.

Broadly, simulation can be broken down into two categories: high-fidelity and low-fidelity. A highfidelity simulator is characterized by realistic out-the-window scene detail, avionics that are cockpitspecific to the aircraft it represents, flight controls with feedback that is representative of actual flight dynamics, and motion platforms. By comparison, low-fidelity simulators are personal computer (PC) based flight simulators that are commonly regarded as games. Low-fidelity simulators are sometimes called PC-based aviation training devices (PCATDs). 
Compared to high-fidelity simulators, low-fidelity simulators are very inexpensive. Yet, investigations have found no differences in training transfer between high- and low-fidelity simulators (Koonce \& Bramble, 1998; Salas, Bowers, and Rhodenizer, 1998). Research suggests that low-fidelity simulation is effective as long as it simulates the cognitive processes required in real world operations; this correspondence is called psychological fidelity (Bowers \& Jentsch, 2001). Incorporating low-fidelity interactive simulation into a computer-based CRM or SRM training program allows for a method of practice. Low-fidelity simulators have been used for some aspects of CRM training in the past, such as practicing aircrew coordination skills. Generally, pilots consider low-fidelity simulators to be an acceptable method of practice (Baker, Prince, Shrestha,Oser, \& Salas, 1993; Brannick, Prince, \& Salas, 2005).

Personal-computer based low-fidelity flight simulators that incorporate peripheral yoke, throttle, and rudder pedals become more than a game and are marketed as a means for pilots to refine their skills. However, the high-bandwidth requirements and the cost of flight control peripherals associated with interactive simulation complicate wide distribution of such a program. Thus there is a need for costeffective scenario-based practice within CRM and SRM that can be widely distributed online, without flight control peripherals.

This investigation assessed a new instructional strategy — guided mental practice (GMP) — as a means of practicing pilot safety concepts online. As an initial investigation into the effectiveness of GMP, this study assessed only one component of CRM and SRM training: situation awareness (SA). Endsley (1995) defined SA as "the perception of the elements in the environment within a volume of time and space, the comprehension of their meaning, and the projection of their status in the near future" (p. 31).

\section{Practice}

\section{Deliberate practice.}

Research has determined that the amount of experience does not directly relate to the acquisition of expert performance. Rather, expert performance is attributable to the amount of deliberate practice accomplished by the learner (van Gog, Ericsson, Rikers, \& Paas, 2005). Deliberate practice, which is carried out to refine or enhance a specific skill, is typically created by an instructor to target specific areas of a task that require improvement. To be effective, deliberate practice activities must be at a challenging level of difficulty, provide feedback, and allow for refinement (van Gog et al., 2005). This type of practice requires a high level of concentration and is effortful to maintain (van Gog et al., 2005).

Although deliberate practice is often included in CRM training programs, it is typically focused on developing conditioned responses rather than on developing higher order thinking skills (Robertson, 2005). It is necessary to design practice scenarios that allow students to practice applying the instructional concepts and to think about how they will react in a real-world situation.

\section{Mental practice.}

Mental practice is characterized by learners deliberately verbalizing or visualizing the information while imagining (Leahy \& Sweller, 2005). Studies on the effect of mental practice come from various disciplines, including sport psychology, cognitive neuroscience, and cognitive psychology (Jackson, Lafleur, Malouin, Richards, \& Doyon, 2001). The instructional strategy of imagining to enhance learning and performance has a long history in the research literature, although the terminology is varied. The terms symbolic rehearsal and imaginary practice originated in the 1930s (Perry, 1939; Sackett, 1934). 
Since that time, the terms mental practice, introspective rehearsal, conceptualization, covert rehearsal, and mental rehearsal have been used to describe the same phenomenon (Driskill, Copper, \& Moran, 1994; Leahy \& Sweller, 2004). Although several studies have evaluated this phenomenon, some with inconclusive results, most indicate that mental practice improves performance (Druckman \& Swets, 1988).

Leahy and Sweller (2004) explained that mental practice is a form of deliberate practice, as the goal of both is to "process material through working memory with the intention of strengthening schemas held in long-term memory" (p. 859). Wiley and Voss (1999) determined that the kind of practice exercise does not matter; it is the mental process it facilitates that results in enhanced learning. Similarly, Jackson et al. (2001) stated that "humans have the ability to generate mental correlates of perceptual and motor events without any triggering external stimulus, a function known as imagery" (p. 1133). This imagery, or mental practice, is a powerful instructional tool for improving performance on both cognitive and psychomotor skills (Driskill et al., 1994). In fact, learners who imagine a concept or procedure often outperform those who study or practice it (Cooper, Tindall-Ford, Chandler, \& Sweller, 2001; Leahy \& Sweller, 2004, 2005).

There are physiological and psychophysical similarities between movements that are physically executed and those that are imagined (Fadiga et al., 1999; Leonardo et al., 1995). Therefore, mental practice improvements are not limited to cognitive tasks but apply to performance on psychomotor tasks as well (Driskill et al., 1994).

\section{Mental practice in education and training.}

There is little published literature on mental practice investigations in the educational domain, whether in training or higher education. The present study is one of the few that addresses the application of mental practice concepts in professional training. Computer assisted mental practice, as utilized in the present investigation, is also rarely addressed in the academic literature. However, a recent investigation developed a virtual reality system to guide a stroke victim through mental practice exercises in which a virtual reconstruction of arm movement was presented. The investigation determined that the device and associated mental practice resulted in significant improvements. The study also concluded that technology-supported mental practice training programs are feasible and potentially effective for improving motor skills (Gaggioli, Meneghini, Morganti, Alcaniz, \& Riva, 2006). GMP, as used in this study, is another example of technology-supported mental practice.

\section{Mental practice theories.}

Sackett's (1934) symbolic learning theory suggests that mental practice improves motor performance through a cognitive rehearsal of task components. This theory is consistent with the findings of several experiments that identify a stronger effect size of mental practice for cognitively demanding tasks (Driskill et al.,1994). However, it does not explain studies that have found an increase in muscle strength after mental practice (Yue \& Cole, 1992). Paivio (1985) proposed another theory of mental practice. According to Paivio's theory, performance improvements resulting from mental practice result from both cognitive and motivational aspects of an activity at several levels. According to a third theory, the benefits associated with mental practice are similar to the benefits associated with self-explanation. Selfexplanation research has found that individuals who effectively problem-solve are likely to explain the material to themselves (Renk1, 1999; Wong, Lawson, \& Keeves, 2002). Cooper et al. (2001) maintained that this phenomenon is an example of mental practice occurring naturally. Self-explanation can be considered a form of deliberate practice that enhances the development of schemas (Ericsson \& Charness, 1994). The literature indicates that the computer-based instructional design community has yet to capitalize on the potential of mental practice. In particular, mental practice may have the potential to 
expand the capabilities of computer-based distance education to enhance complex and highly technical skills.

\section{METHOD}

\section{Training Program}

For the present investigation, a 90 minute online training program was created. The training program focused on SRM safety skills, including situation awareness. Within the 90 minutes of training, several low-fidelity simulator scenarios were integrated to provide learners with the opportunity to practice SRM concepts. Two identical versions of the training program were created, with identical practice scenarios. The only difference between the two versions of training was whether students were able to control the scenario hands-on or to observe a video of the flight scenario for GMP (see Figure 1).

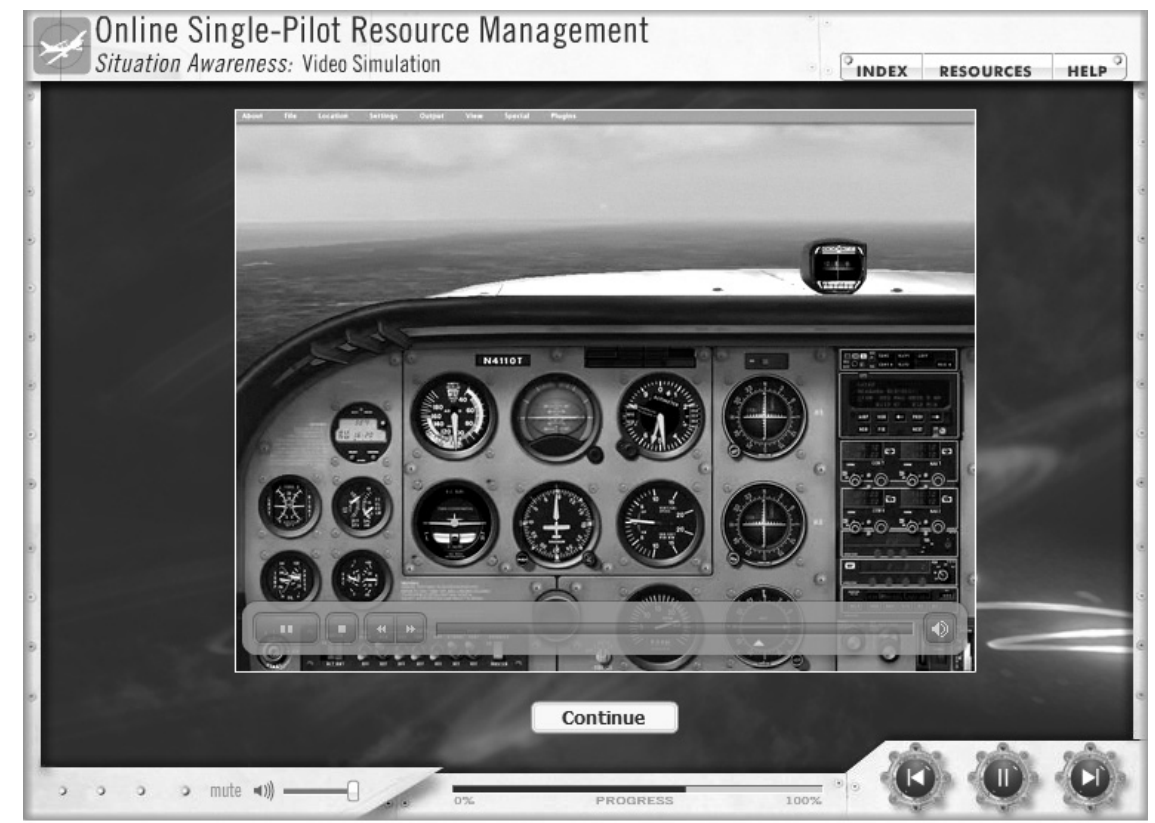

Figure 1. A screenshot of the flight scenario video used for hands-on practice and GMP of situation awareness skills

The first version of training allowed students to have complete control over the aircraft within the practice scenarios through the use of peripheral yoke and rudder pedals. The experimental group that completed this version of training was referred to as the hands-on practice condition.

The second version of training presented students with videos of flight scenarios, which they were not able to interact with or control. Students who completed the second version of training were asked to imagine themselves as the pilot of the flight as they watched the scenario video. The experimental group that completed this version of training was referred to as the GMP condition. GMP differs from traditional mental practice, which is typically an entirely internal process, in that the training course guides the learner through the mental practice exercise. 


\section{Participants}

The sample comprised a total 36 of individuals. There were 32 males $(88.9 \%)$ and 4 females $(11.1 \%)$. The age range of the participants was 18 to 31 (mean $=22.14, S D=3.04$ ). Although most participants were at the private pilot level, flight hour experience ranged from 50 hours to 825 hours (mean $=164.8$, $S D=166.1$. All participants were required to have achieved a minimum of a private pilot certificate. Participants were recruited through an email distributed to alumni, students, and associates of the aviation program at the university and college associated with this investigation. A recruitment email was also distributed to several pilot clubs and associations in the local area.

In order to possess a pilot's license individuals must complete a medical evaluation. Therefore, physical and mental disabilities, which are important considerations in the design and development of most online learning programs, were not of significant concern in the investigation.

\section{Procedure}

This study utilized an experimental one-variable multiple condition control group design. The independent variable (IV) was SRM training. Three groups of 12 participants were formed in the study: (a) those who received training with hands-on practice, (b) those who received training with GMP, and (c) a control group that received no training. The dependent variable was situation awareness performance, as measured by the situation awareness global assessment technique (SAGAT) within a high-fidelity flight simulator post training (Endsley 1995, 2000). The SAGAT is a commonly used assessment of pilot situational awareness (Endsley 2000).

Data collection took place over a 2-week period. The involvement of individual participants was completed in a single day and was approximately 3 hours in duration. One at a time, participants arrived at the training facility and were randomly assigned to a treatment group. Participants within experimental groups then completed a 90 minute SRM training course. Hands-on and GMP training took place in separate quiet rooms. Participants in the hands-on practice condition completed training at a computer equipped with peripheral keyboard and mouse devices used to control the training program's multimedia presentation and yoke and rudder pedals to control the low-fidelity flight scenarios, as pictured in Figure 2. Participants in the GMP condition completed training at a computer with only keyboard and mouse peripherals.

Following training, participants were asked to plan a cross country flight which would be completed in a high-fidelity Cessna 172 flight simulator. Participants were provided with planning materials, including a description of the route and intention of the trip, a map, flight computer, plotter, weather, and blank paper for notes. Flight planning was also completed in isolation, in a quiet room. Participants were given an open-ended amount of time to complete their flight planning and were asked to notify the researcher when finished (the amount of time used ranged from a few minutes to over an hour). Participants in the control group did not complete any computer-based SRM training and were immediately given planning materials for the cross country trip in the high-fidelity flight simulator.

Once participants notified the researcher that they had completed flight planning they were given a SAGAT briefing, which explained how the simulator would be paused at random intervals and questions would be asked by the researcher. It was explained that the researcher would compare the participant's answers to the current state of the flight to generate quantitative data regarding situation awareness. Participants were asked to respond to all questions to the best of their ability and as quickly as possible. Following the SAGAT briefing, participants were escorted to the Cessna 172 high-fidelity flight simulator to complete their cross country exercise. The high-fidelity simulator was operated by a 
pilot/research assistant and observed by a researcher. Once the cross country flight was finished, the participants were debriefed.

The SAGAT queries were developed before conducting the study. To evaluate performance, the researcher compared the participant's answers to the status of the flight simulator to generate quantitative data describing the participants' performance. A score of one was given for a correct response and zero for an incorrect response.

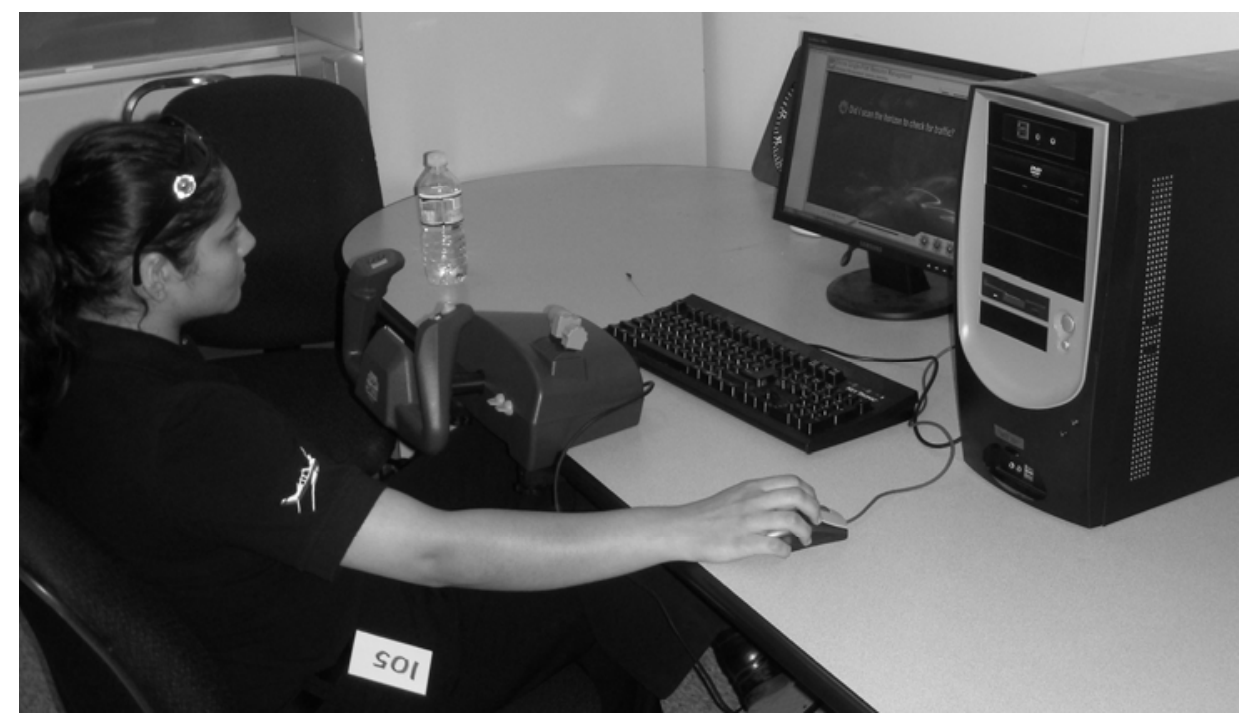

Figure 2. Peripheral yoke and rudder pedals used within the hands-on practice condition

\section{RESULTS}

Performances of individuals in the sample were measured using the SAGAT (Table 1). A univariate analysis (that is, without controlling for age, gender and other variables that might have an effect on the performance measures), identified significant differences across groups at the 0.05 level for SAGAT $(p=$ 0.001). In order to assess the nature of the group differences, a post hoc Least Significant Difference procedure was performed. This procedure allowed performing multiple pairwise comparisons in order to determine which groups had significantly higher or lower scores than others.

Table 1. Differences in Performance Measures by Group

\begin{tabular}{ccc}
\hline Group & & SAGAT \\
\hline Hands-on practice & Mean & 13.08 \\
& $S D$ & 2.35 \\
Mental practice & Mean & 13.50 \\
& $S D$ & 2.43 \\
Control & Mean & 10.00 \\
& $S D$ & 2.22 \\
\hline Total & Mean & 12.19 \\
& $S D$ & 2.77 \\
\hline
\end{tabular}

Note. The $p$ value for this table corresponds to one-way ANOVA using SAGAT as the dependent variable and Group as the independent variable. $p=.001$ 
For the SAGAT variable, it was found that the control group had lower scores than the GMP ( $p=$ $0.001)$ and hands-on practice $(p=0.003)$ groups. However, no significant differences were found between the GMP and hands-on groups $(p=0.665)$. These findings provide evidence that safety training with GMP and safety training with hands-on practice have approximately the same degree of effectiveness in increasing SAGAT scores.

\section{SUMMARY AND DISCUSSION}

The results of this study indicate that guided mental practice was as effective as hands-on practice in improving situation awareness skills, as a component of CRM or SRM training programs. These findings promote GMP as a feasible and effective instructional strategy for computer-based training of nontechnical pilot skills within CRM and SRM. In addition, the incorporation of GMP can ameliorate the problem of distributing peripheral yoke and rudder pedals for the control of low-fidelity simulators within online pilot safety training.

The findings of this investigation are relevant to the broader academic and instructional design community for two main reasons. First, the lack of significant difference between the hands-on and mental practice conditions supports the hypothesis that both GMP and hands-on practice are effective deliberate practice methods within pilot safety training. Second, the significant performance improvement demonstrated by the groups that completed training (incorporating either hands-on or GMP), compared to the group that did not complete training, supports the claim that asynchronous computer-based training is a feasible option for delivering non-technical training.

These results suggest that guided mental practice is a promising e-learning instructional strategy. However, because of the infancy of this approach, additional work is required to assess its effectiveness in delivering other CRM and SRM concepts beyond situation awareness. Finally, it is recommended that future research assess long term changes in behavior and organizational impacts associated with computer-based pilot safety training. 


\section{REFERENCES}

Baker, D., Prince, C., Shrestha, L., Oser, R., \& Salas, E. (1993) Aviation computer games for crew resouce management training. The International Journal of Aviation Psychology, 3, 143-156.

Bowers, C.A., \& Jentsch, F. (2001) Use of commercial, off-the-shelf, simulations for team research. In Advances in human performance (ed E. Salas), 293-317. Amsterdam, NL: Elsevier Science.

Brannick, M. T., Prince, C., \& Salas, E. (2005) Can PC-based systems enhance teamwork in the cockpit? The International Journal of Aviation Psychology, 15(2), 173-187.

Cooper, G., Tindall-Ford, S., Chandler, P., \& Sweller, J. (2001) Learning by imagining. Journal of Experimental Psychology: Applied, 7, 68-82.

Driskill, J.E., Copper, C., \& Moran, A. (1994) Does mental practice enhance performance? Journal of Applied Psychology 79, 481-492.

Druckman, D. \& Swets, J. (1988) Enhancing human performance. Washington, DC: National Academy Press.

Endsley, M.R. (1995) Measurement of situation awareness in dynamic systems. Human Factors, 37, 6584.

Endsley, M.R. (2000) Direct measurement of situation awareness: validity and use of SAGAT. In Situation Awareness Analysis and Measurement (eds M.R. Endsley \& D.J. Garland), 147-173. Mahwah, NJ: Erlbaum.

Ericsson, K.A., \& Charness, N. (1994) Expert performance: its structure and acquisition. American Psychologist, 49, 725-747.

Fadiga, L., Buccino, G., Craighero, L., Fogassi, L., Gallese, V., \& Pavesi, G. (1999) Corticospinal excitability is specifically modulated by motor imagery: a magnetic stimulation study. Neuropsychologia, 37, 147-158.

Gaggioli, A., Meneghini, A., Morganti, F., Alcaniz, M., \& Riva, G. (2006) A strategy for computerassisted mental practice in stroke rehabilitation. Neurorehabilitation and Neural Repair, 20, 503-507.

Helmreich, R.L., Merritt, A.C., \& Wilhelm, J.A. (1999) The evolution of crew resource management training in commercial aviation. The International Journal of Aviation Psychology, 9, 19-32.

Jackson, P.L., Lafleur, M.F., Malouin, F., Richards, C., \& Doyon, J. (2001) Potential role of mental practice using motor imagery in neurologic rehabilitation. Archives of Physical Medical Rehabilitation, 82, 1133-1141.

Jensen, R.S. (1995) Pilot judgement and crew resource management. Burlington, VT: Ashgate. Koonce, J.M., \& Bramble, W.J. Jr. (1998) Personal computer-based flight training devices. The International Journal of Aviation Psychology, 8, 277-292. 
Leahy, W., \& Sweller, J. (2004) Cognitive load and the imagination effect. Applied Cognitive Psychology, 18, 857-875.

Leahy W., \& Sweller, J. (2005) Interactions among the imagination, expertise reversal, and element interactivity effects. Journal of Experimental Psychology: Applied, 11(4), 266-276.

Leonardo, M., Fieldman, J., Sadato, N., Campbell, G., Ibanez, V., \& Cohen, L. (1995) A magnetic resonance functional neuroimaging study of cortical regions associated wtih motor task execution and motor ideation in humans. Human Brain Mapping, 3, 83-92.

Paivio, A. (1985) Cognitive and motivational functions of imagery in human performance. Canadian Journal of Applied Sport Sciences, 10, 22S-8S.

Perry, H.M. (1939) The relative efficiency of actual and imaginary practice in five selected tasks. Archives of Psychology, 34, 5-75.

Renkl, A. (1999) Learning mathematics from worked-out examples: analyzing and fostering selfexplanation. European Journal of Psychology of Education, 14, 477-488.

Robertson, C.L. (2005) Development and transfer of higher order thinking skills in pilots. Ph.D. dissertation, Capella University. Retrieved 04/10/2008 from Proquest Digital Dissertations database.

Sackett, R.S. (1934) The influence of symbolic rehearsal on the retention of a maze habit. Journal of General Psychology, 10, 376-395.

Salas, E., Bowers, C.A., \& Rhodenizer, L. (1998) It is not how much you have but how you use it: toward a rational use of simulation to support aviation training. The International Journal of Aviation Psychology, 8, 197-208.

Salas, E., Rhodenizer, L., \& Bowers, C.A. (2000) The design and delivery of crew resource management training: Exploiting available resources. Human Factors, 42, 490-512.

van Gog, T., Ericsson, K.A., Rikers, R.M., \& Paas F. (2005) Instructional design for advanced learners: Establishing connections between the theoretical frameworks of cognitive load and deliberate practice. Educational Technology Research and Development, 53(3), 73-81.

Wiley, J., \& Voss, J.F. (1999) Constructing arguments from multiple sources: Tasks that promote understanding and not just memory for text. Journal of Educational Psychology, 91, 301-311.

Wong, R.M., Lawson, M.J., \& Keeves, J. (2002) The effects of self-explanation training on students' problem-solving in high-school mathematics. Learning and Instruction, 12, 233-262.

Yue, G., \& Cole, K.J. (1992) Strength increases from the motor program comparison of training with maximal voluntary and imagined muscle contractions. Journal of Neurophysiology, 67, 1114-1123. 